\title{
The Application of Thinking Aloud Method in the Listening and Speaking Teaching of College English
}

\author{
Zhai Man \\ School of Foreign Languages \\ Jilin Business and Technology College \\ Changchun, China \\ zhaiman@126.com
}

\author{
Mou Xingui \\ Foreign Language Department, the Fundamental Base \\ Aviation University of Air Force \\ Changchun, China \\ 1349473117@qq.com
}

\begin{abstract}
Thinking aloud method has been used to describe and study the cognitive evolution of human beings in the process of dealing with problems. Thinking aloud means speaks out the immediate thoughts and all the information in the minds at any time and place when people are engaged in a task, which can also be understood as the behavior of using voice to express the state of mind. This paper adopts the thinking-aloud method to describe students' cognitive process, comparative analysis process of problem solving, the thinking and judgment process in the college English listening and speaking course, which helps to further improve and optimize the teaching methods and improve the students' English comprehensive ability.
\end{abstract}

Keywords-thinking aloud method; college English course; learning strategy; listening and speaking teaching

\section{INTRODUCTION}

As a kind of investigation method, the thinking aloud method is widely used in the study of a specific human thinking process, which can directly reflect the working state of the brain, the mode of thinking and the thinking process. The application of the thinking aloud method in College English teaching can assist teachers to understand students' learning process, grasp students' mode of thinking and obtain true feedbacks so as to examine the teaching effect and guide the teaching method ${ }^{[2]}$. The classroom teaching of college English includes the reading and writing course and the listening and speaking course. The thinking-aloud method, as an effective and innovative teaching method, is used in the listening and speaking classroom teaching of College English, which helps and promotes the listening and speaking teaching to some extent.

\section{RELATIONSHIP BETWEEN LISTENING AND SPEAKING AND THINKING ALOUD METHOD}

\section{A. Interrelationship Between Listening and Speaking Courses}

Listening comprehension is the most important and the most difficult skill of the five basic abilities in foreign language learning, in other words, listening, speaking, reading, writing and translating. However, the study of listening teaching method is relatively lagging compared with the other four skills Because of the lack of visibility of the listening process, the research method is difficult to determine, which lead to the lack of effective guidance for the foreign language learning students especially the English majors whose overall listening level is generally not high ${ }^{[7]}$. It is believed that the key to improve people's listening level is to explore the cognitive processes or cognitive behavior while they are completing the listening tasks. In simple terms, the key point is to find out how students listen to the problem in the process of listening comprehension and what kind of method is used to solve the problem when they meet up with it. The emergence of the think-aloud method provides a new way of thinking and also an effective way to sort out the problems mentioned above.

Among the five English learning skills of listening, speaking, reading, writing and translating, listening and speaking are the prerequisites and foundation for improving English skills. The present college English textbook is composed of two parts, that is, the reading and writing part and listening and speaking part, from which we can see that the listening and speaking part plays an important role in College English teaching. With the multi intervention of new media and the development of Internet plus era, more and more attention has been paid to the teaching mode of the integration of listening and speaking, which can also promote reading and writing skills and form the learning effect of organic combination and complementing each other. There is no separation between listening and speaking ${ }^{[1]}$. To listen is to comprehend and to speak is to express. Listening and speaking is the basic and the most direct and effective way to learn the language and the cultural exchanges between different cultures. College English Teaching abandons the shortcomings of the neglect of listening and speaking ability of the examination oriented education in senior high school English teaching, and increases the proportion of listening and speaking lessons. However, the listening and speaking process especially the cognitive process of listening problem-solving path is usually easy to be neglected. The thinking-aloud method record the longitudinal cognitive analysis process to a proper extent, which can demonstrate problems and explain the process of understanding and remembering revising so as to guide the correction, enhancement and improvement of the teaching behaviors. 


\section{B. Reconsideration on Thinking Aloud Method}

The think-aloud or think-aloud recording method requires the subjects to express their immediate thoughts in completing the process of a specific task ${ }^{[6]}$. The testers record it by the way of recording and finally transfer it into the text form, whose characteristics are suitable for small sample surveys. As one of the most important research methods in cognitive psychology and educational psychology, thinking aloud is also widely used in foreign language teaching. Take the listening teaching research as an example, surveys based on the method of thinking aloud are increasingly used in many aspects such as the listening process research, strategy research and the influence factors research, which can contribute to our scientific understanding of the psychological process of listening comprehension and our fully understanding of the foreign language learning groups (individuals)'s the application situation to all kinds of listening strategies. At present, China's foreign language educational circles is gradually applying the thinking aloud method to the listening comprehension and strategy research, but the overall number of research is very limited, and the thinking aloud research specifically used for the teaching of English listening and speaking is less.

\section{THINKING-ALOUD METHOD AND COGNITIVE PROCESS OF LISTENING AND SPEAKING}

\section{A. Operation Process}

To apply thinking-aloud research method is capable of comprehending the explicit procedure of macroscopic listening and speaking cognitive process. Specifically speaking, thinking-aloud research method could help test the acceptation conditions on speech sounds information of learners. Furthermore, it can also assist to analyze the distribution conditions of learners' concentrations. And proceed to detailed information about learners' understanding, analyzing, estimating on language messages. The reaction situation would be also displayed through research process. At the same time, thinking-aloud research method is adopted for studying the inner relationship and influences among individual steps. Comprehension is determined by reception. One could enter into understanding process only after accurate reception. Meanwhile, comprehension counter reflects reception. In certain perspectives, comprehension shows directions and set expectation values for reception. The diversity differentia of different learners belonging to different levels could be exhibited $^{[3]}$. Moreover, it enables the metrical explore the relationship among language, pragmatics and cognitive process in listening and speaking, and students' understanding, using and cognation treatment on language are explained and shown by research process.

1) Determination of subjects and materials: The subjects are the first grade students of non-English Majors (group 1) and students from experimental classes of the same grade (group 2). Students in group 1 have a lower English level and students in group 2 have a higher level of English. Both of them have not participated in CET 4 yet. The test questions are selected from Book 2 of New Horizon College English listening and Speaking (Foreign Language Teaching and Research Press, 2015), and which the subjects have never been exposed to and match the English level of freshmen in college. The subjects were recorded one by one without interference in the language lab. After the test paper is sent to the subjects, the listening will be played, which consists of a long dialogue, and 5 multiple-choice questions. The topic of oral English is to express the advantages and disadvantages. After listening, we will start the thinking aloud recording and ask the subjects to speak out the problem-solving process and the psychological feelings. Give two minutes to the subjects before the colloquial expression, and also record their thinking aloud. Finally, we get 2 audio files that contain the process of listening comprehension, the process of oral thinking and the process of oral presentation.

2) Process analysis: The cognitive and problem-solving process in College English listening classes consists of the preparation period, that is, subjects' topic reading process, and the speech input and segmentation process, during which there will exist phenomenon of information loss because of liaison, ellipsis, accent and other factors; After the code is saved, it opens the top-down understanding process of knowledge base - semantic analysis - word recognition and bottom-up word recognition- meaning analysis, which is one of the stage that can reflect the cognitive process most. The results of the thinking aloud method showed that the using rate of the bottom-up in the process was much higher than the word recognition based on knowledge base, which showed that the students failed to promptly extract the related background knowledge. The following process of information arrangement and preservation cannot be recorded directly in the thinking aloud. The process of analysis, matching, judgment, exclusion and conclusion is another very important segment of the sound recording of the cognitive process, which can reflect the subjects 'logical reasoning ability, strategy selection and problem-solving skills, and the process of semantic and discourse analysis. During the following oral English test preparation time, the two groups of subjects all use their mother tongue at the beginning and they all have showed clear thinking and clear expression at this time but have showed varying degrees of pause and unclear expression during the English transformation process ${ }^{[4]}$. The thinking aloud records showed that the former met with problems of unfamiliar words while the latter expressed grammatical disorder. In the process of formal oral expression, subjects in group one only speak out some incomplete words and phrases while subjects from group two only uses simple sentences.

\section{B. Research Results}

The results show that subjects from group two have better accuracy for two questions that have no direct answers from the original passage. Subjects with different levels of English have all used the four listening strategies, that is, the surface semantic matching, the deep semantic matching, background knowledge and reasoning. The surface semantic matching can help the subjects to solve the less difficult problems in a short period of time, and the deep semantic matching can help the subjects to answer the higher level questions. After integrating some subjective thinking and problem-solving skills into the activating background knowledge and logical reasoning process, the research results of the thinking aloud method 
reflected that subjects with higher English level can accurately extract the relevant information from the brain to effectively assist the problem solving. And they can effectively control the subjective judgment, subtly utilize the answering skills in the process of reasoning, focus on the capturing of session details and speculate unknown information by using the known information so as to achieve better listening effect and higher accuracy of problem solving .In the following dictation process, thinking aloud shows that the subjects' thinking expression are very clear. The reason that causes short dictation from subjects of group two is the lack of sentence pattern and improper collocation $^{[5]}$. Thus, we should strengthen background knowledge input in College English listening and speaking course, train the students' problem solving skills and reasoning skills and increase their vocabulary and sentence patterns so as to enhance students' listening and speaking ability.

\section{CONCLUSION}

The investigation method of thinking aloud method plays a unique role in foreign language teaching and learning and involves many branches of foreign language teaching and learning, which is widely, used in the College English listening and speaking teaching courses. With the in-depth development of the study of College English Teaching in the field of cognitive linguistics and the interdisciplinary research of neuroscience, the application of thinking aloud in the listening and speaking class give full play to its unique function and role, whose sufficient theoretical significance and application value draw the attention of more and more researchers. The application of the thinking aloud method in listening and speaking can help teachers to improve the learners' learning methods and strategies, which can help to do the listening and speaking training so as to improve their English listening and speaking ability.

\section{REFERENCES}

[1] Anderson, J.R. Cognitive Psychology and Its Implication [M]. New York: W.H. Freeman, 1985.

[2] Barkaoui K. Think-aloud protocols in research on essay rating: An empirical study of their veridicality and reactivity [J]. Language Testing, 2011(1):51-75.

[3] Bowles M.A. The Think-Aloud Controversy in Second Language Research [M]. New York: Routledge, 2010.

[4] Leow R.P. \& Morgan-Short K. To think aloud or not to think aloud: The issue of reactivity in SLA research methodology [J]. Studies in Second Language Acquisition, 2004(1):35-57.

[5] Monacelli, C. Self-preservation in Simultaneous Interpreting [M]. Amsterdam: John Benjamins,2009.

[6] Nida, E.A. Language, Culture and Translation [M]. Shanghai:Shanghai Foreign Language Education Press, 1993: 109-110.

[7] Sanz C, Lin H.J., Lado B, Bowden H.W. \& Stafford C.A. Concurrent verbalizations, pedagogical conditions, and reactivity: Two CALL studies [J]. Language Learning, 2009(1):33-71. 